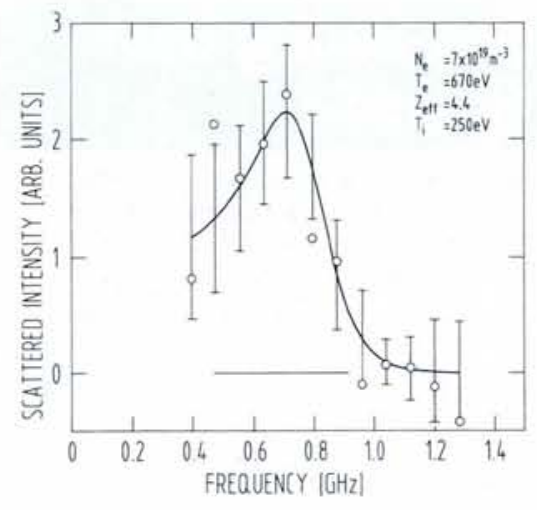

Fig. 9-Measured scattering spectrum for a He plasma with an electron density $N_{e}$ of $7 \times 10^{19} \mathrm{~m}^{-3}$ recorded using a single laser pulse.

$\left(<10^{-18} \mathrm{~W} / \mathrm{Hz}\right)$. The heterodyne technique however, offers several unique opportunities:

- Sensitivity close to the quantum noise limit.

- Rapid detection for laser pulses lasting about 1 us.

- Insensitivity to the noisy tokamak environment.

Successful experiments have recently been carried out by research groups at the Ecole Polytechnique Fédérale in Lausanne and at the University of Düsseldorf using a pulsed $\mathrm{D}_{2} \mathrm{O}$ laser at $385 \mu \mathrm{m}$. A single laser shot was found to be sufficient to determine the ion temperature in $\mathrm{H}, \mathrm{D}$ and $\mathrm{He}$ plasmas with electron densities $N_{e}$ above $5 \times$ $10^{19} \mathrm{~m}^{-3}$ (Fig. 9).

\section{Conclusions}

Since its introduction in astronomy, the sub-millimetre/far infrared heterodyne spectrometer has come to be widely used in other, very different, fields of research. A feature that allowed this extension was the demonstration of an airborne system that had proved itself in a hostile environment. Expected improvements in sensitivity, bandwidth and reliability combined with the capacity to operate at wavelengths smaller than $100 \mu \mathrm{m}$ ensure an exciting future.

\section{FURTHER READING}

Astronomy:

Schmid-Burgk J. et al., Astron. and Astrophys. 215 (1989) 150.

Röser H.P. and Schmid-Burgk J., Sterne und Weltraum 28 (1989) 648

Atmospheric Physics:

Wattenbach R. et al., J. Geophys. Res. 89 , No. D5 (1984) 7285.

Plasma Physics:

Behn R. et al., Phys. Rev. Lett. 62 (1989) 2833.

Sub-mm/FIR techniques:

Chantry G.W., Long-Wave Optics, Vols 1 and 2 (Academic Press, London) 1984.

\title{
1990 Nobel Prize in Physics
}

The 1990 Nobel Prize in Physics has been awarded jointly to Professors Jerome Friedman and Henry Kendall, both of the Massachusetts Institute of Technology, Cambridge, MA, USA, and Richard Taylor of Stanford University, Stanford, CA, USA for their pioneering investigations of deep inelastic scattering of electrons on protons and bound neutrons which have been of essential importance for the development of the quark model in particle physics.

The three prizewinners were key members of the SLAC-MIT team which confirmed in 1968 clear signs that there exists an inner structure in the proton and neutron of the atomic nucleus. By 1972, interpretation of their results in terms of quarks was assured and work on neutrino scattering started to provide supporting evidence. Their findings therefore paved the way towards today's understanding of the constituents of matter.

An appreciation will be published next month.

\section{Acusto-Optical Spectrometers with Proven Stability}

Elson Research Inc. designs and manufactures acusto-optical spectrum analyzers (AOS) specifically for research applications in radio astronomy or atmospheric physics.

A unique active frequency stabilization technique has earned Elson AOS analyzers a reputation for high sensitivity and excellent reliability.

Each Elson AOS analyzer is tailormade for your specific application; bandwidths available range from 40 $\mathrm{MHz}$ to $1.5 \mathrm{GHz}$, with resolutions from $50 \mathrm{kHz}$ to $10 \mathrm{MHz}$. A subsystem comprises:

* Acusto-optical processor

* Electronics unit

* RF unit

* All power supplies and controls

A subsystem can actually combine up to four spectrometers in a compact chassis for a standard 19" racka rugged, space-saving design which allows easy transportation, installation and maintenance.

When compared to a 1024-channel filter bank for atmospheric research, an Elson AOS offers both wide and narrow resolutions for faster analysis, avoids the need for multiplexing and does not suffer from defective channels. It is also significantly cheaper to purchase and maintain.

The world's leading supplier of commercial AOS analyzers since 1985, Elson Research's recent installations include: The James Clerk Maxwell Telescope in Hawaii (1988), the University of Helsinki (1989) and, for atmospheric physics, the Helsinki University of Technology (double system, 1990).

Elson Research arranges orientation courses, user-training, service-training and maintenance world wide. And ongoing research allows your subsystem to be continually upgraded to the stateof-the-art level.

For more information, please mail or fax Dr. Lauri Malkamäki at:

Elson Research Inc.

P.O. Box 167

Salem, NH 03079-9998

Fax: 603-893-2131

\section{ELSON}

I am interested in reliable AOS analyzers, please send me the following information:

Elson AOS-series specifications

Theory and principles of AOS operation (ISBN 952-90-1704-9)

Application: $\square$ Astronomy $\square$ Atmospheric Physics $\square$ Other

Name and title:

Institute:

Address: 